\title{
Beginn der Dignitätsprüfung im Bereich der Unfall-, Militär- und Invalidenversicherung
}

Wie bereits vor einiger Zeit informiert, haben die Tarifparteien FMH, H+ und Versicherer UV/MV/IV vereinbart, die Prüfung von Besitzstands- und Dignitätsdaten in Form von Einzelabfragen der FMH-Dignitätsdatenbank durchzuführen. Diese Vereinbarung tritt nun wie folgt in Kraft und wird dabei in zeitlichen Etappen operativ umgesetzt:

- seit 1. Januar 2008: Beginn der Dignitätsprüfung für Leistungen von Ärzten in freier Praxis;

- im 1. Quartal 2008: Beginn der Dignitätsprüfung für Leistungen von Spitalärzten;

- ab 2. Quartal 2008: Dignitätsprüfung mit möglicher Rückweisung von nicht dignitäts- konformen Leistungen von Ärzten in freier Praxis;

- ab 3. Quartal 2008: Dignitätsprüfung mit möglicher Rückweisung von nicht dignitätskonformen Leistungen von Spitalärzten.

Dabei erfolgt die Prüfung nicht durch die einzelnen Versicherer, sondern in deren Auftrag durch die Zentralstelle für Medizinaltarife UVG (ZMT).

Mit Einführung der Dignitätsprüfung im UV/MV/IV-Bereich ab 1. Januar 2008 wird eine wesentliche, vertraglich vereinbarte Massnahme zur Sicherstellung der Qualität von ärztlichen Leistungen hinsichtlich der Komponente Aus-, Fort- und Weiterbildung umgesetzt.

\section{Début de la phase de vérification des valeurs intrinsèques dans le domaine de l'assurance-accidents, de l'assurance- invalidité et de l'assurance militaire}

Comme nous l'avions annoncé il y a quelque temps, les partenaires tarifaires $\mathrm{FMH}, \mathrm{H}+$ et les assureurs du domaine AA/AM/AI ont convenu de procéder à des vérifications des droits acquis et des valeurs intrinsèques sur la base des données individuelles des médecins figurant dans la banque de données des valeurs intrinsèques de la FMH. La convention conclue entre en vigueur en 2008 et sera appliquée par étapes selon le calendrier suivant:

- depuis le $1^{\text {er }}$ janvier 2008: début de la vérification des valeurs intrinsèques des médecins en pratique privée;

- premier trimestre 2008: début de la vérification des valeurs intrinsèques des médecins hospitaliers;

- dès le deuxième trimestre 2008: vérification des valeurs intrinsèques avec éventuel refus des prestations non conformes fournies par les médecins en pratique privée;

- dès le troisième trimestre 2008: vérification des valeurs intrinsèques avec éventuel refus des prestations non conformes fournies par les médecins hospitaliers.

Les assureurs ne procèdent pas eux-mêmes à cette vérification. Ils ont mandaté pour ce faire le Service central des tarifs médicaux LAA (SCTM).

Le lancement au $1^{\text {er }}$ janvier 2008 de cette vérification est l'application concrète d'une mesure contractuelle essentielle visant à garantir la qualité des prestations médicales au niveau de la formation prégraduée, postgraduée et continue. 